\title{
Effect of hot forging on the microstructure and superplastic properties of the powder nickel base superalloy EP741NP
}

\author{
A. A. Ganeev ${ }^{\dagger}$, V.A. Valitov, M. I. Nagimov, V.M. Imayev \\ †artem@imsp.ru \\ Institute for Metals Superplasticity Problems RAS, 39 S. Khalturin St., Ufa, 450001, Russia
}

\begin{abstract}
The work is devoted to the study of the microstructure and superplastic properties of the nickel base powder superalloy EP741NP subjected to different hot forging procedures, which were performed after preliminary heterogenization treatment of the initial HIP-ed material. Condition 1 of the superalloy was obtained through three-stage uniaxial canned forging with intermediate recrystallization annealing at a temperature slightly lower than the $\gamma^{\prime}$ solvus temperature $\left(T_{s}-30^{\circ} \mathrm{C}\right.$, where $T_{s}$ is the $\gamma^{\prime}$ solvus temperature). Some of the forged workpieces were subjected to additional low-temperature uniaxial forging under isothermal conditions at the temperature of $T_{s}-230^{\circ} \mathrm{C}$ (condition 2). The produced superalloy conditions were studied by scanning electron microscopy, in particular, using electron backscatter diffraction (EBSD) technique. In condition 1 , predominantly a fine-grained microstructure of the duplex type was obtained. Condition 2 contained an ultrafine-grained constituent with a size of $\gamma$ grains $/ \gamma^{\prime}$ particles less than $1 \mu \mathrm{m}$ resulted from recrystallization processes at a lower temperature. It was revealed that condition 2 demonstrated superplastic elongations $(\delta=200-1320 \%)$ at temperatures $T=900-1000^{\circ} \mathrm{C}$ $\left(\dot{\varepsilon}=5 \times 10^{-4}-10^{-3} \mathrm{~s}^{-1}\right)$, whereas condition 1 showed superplastic elongations $(\delta=200-630 \%)$ at significantly higher temperatures $\left(T=1075-1125^{\circ} \mathrm{C}, \dot{\varepsilon}=5 \times 10^{-4}-10^{-3} \mathrm{~s}^{-1}\right)$. The decrease in the superplastic temperatures and the increase in the superplastic elongations in condition 2 (in contrast to condition 1) was caused by: i) a larger length of high-angle grain and interphase boundaries that was favorable for operating grain boundary sliding, which is the main mechanism of superplastic deformation; ii) higher thermal stability of the microstructure providing small changes in the $\gamma$ and $\gamma^{\prime}$ sizes during superplastic flow; iii) a smaller fraction of twin boundaries, which are unfavorable for grain boundary sliding.
\end{abstract}

Keywords: nickel base superalloys, hot forging, microstructure, superplastic properties.

УДК: 669.245, 621.7.043

\section{Влияние деформационно-термической обработки на микроструктуру и сверхпластические свойства порошкового никелевого сплава ЭП741НП}

\author{
Ганеев А. А. ${ }^{\dagger}$ Валитов В. А., Нагимов М.И., Имаев В. М. \\ Институт проблем сверхпластичности металлов РАН, ул. С. Халтурина, 39, Уфа, 450001, Россия
}

Работа посвящена исследованию микроструктуры и сверхпластических (СП) свойств жаропрочного порошкового никелевого сплава ЭП741НП, подвергнутого разной деформационно-термической обработке (ДТО), выполненной после предварительной гетерогенизационной обработки исходного компактированного сплава. Состояние 1 сплава было получено с помощью трехстадийной одноосной деформации сжатием в стальном контейнере с промежуточными рекристаллизационными отжигами при температуре немного ниже температуры полного растворения $\gamma^{\prime}$ фазы $\left(T_{s}-30^{\circ} \mathrm{C}\right.$, где $T_{s}-$ температура полного растворения $\gamma^{\prime}$ фазы). Часть заготовок была подвергнута дополнительной низкотемпературной деформационной обработке, которую проводили в изотермических условиях сжатием без контейнера при температуре $T_{s}-230^{\circ} \mathrm{C}$ (состояние 2). Полученные состояния сплава изучали с помощью сканирующей электронной микроскопии, в том числе методом дифракции обратно-рассеянных электронов (EBSD-анализа). В заготовках сплава в состоянии 1 была получена преимущественно мелкозернистая структура типа микродуплекс. Заготовки сплава в состоянии 2 содержали ультрамелкозернистую составляющую с размером зерен/частиц менее 1 мкм, образовавшуюся в результате развития рекристаллизационных процессов при пониженной температуре. Обнаружено, что образцы сплава в состоянии 2 демонстрируют СП удлинения $(\delta=200-1320 \%)$ при температурах $T=900-1000^{\circ} \mathrm{C}\left(\dot{\varepsilon}=5 \times 10^{-4}-10^{-3} \mathrm{c}^{-1}\right)$, тогда как в состоянии 1 образцы сплава 
показывают СП удлинения $(\delta=200-630 \%)$ при значительно более высоких температурах $\left(T=1075-1125^{\circ} \mathrm{C}\right.$, $\left.\dot{\varepsilon}=5 \times 10^{-4}-10^{-3} \mathrm{c}^{-1}\right)$. Снижение температуры проявления эффекта СП и повышение СП свойств в состоянии 2 сплава (в сравнении с состоянием 1) обусловлено: і) увеличением протяженности высокоугловых межзеренных и межфазных границ, что благоприятствовало развитию зернограничного проскальзывания - основного механизма СП деформации; ii) высокой стабильностью микроструктуры - слабым изменением размера $\gamma$ зерен и $\gamma^{\prime}$ частиц в процессе СП течения; iii) уменьшением доли двойниковых границ, неблагоприятных для развития зернограничного проскальзывания.

Ключевые слова: жаропрочные никелевые сплавы, деформационно-термическая обработка, микроструктура, сверхпластические свойства.

\section{1. Введение}

В качестве материала дисков и других вращающихся деталей для газотурбинных двигателей (ГТД) широко используют высоколегированные жаропрочные никелевые сплавы [1], изготавливаемые с помощью порошковых технологий, исключающих возникновение макро- и микросегрегаций, характерных для слитков таких сплавов [2]. Технологии изготовления, как правило, основываются на использовании горячего изостатического прессования (ГИП), с помощью которого получают деталь или полуфабрикат требуемой формы [2,3]. Между тем для достижения улучшенных механических (эксплуатационных) свойств, а также обеспечения стабильности гарантированного уровня свойств представляет интерес подвергать компактированный материал горячей экструзии и/или изотермической штамповке [4-8]. При этом для повышения выхода годного металла при изготовлении сложнопрофильных деталей типа «диск», учитывая высокую стоимость высоколегированных никелевых сплавов, перспективной является технология, включающая изотермическую штамповку и/или раскатку в СП условиях $[9,10]$. Для реализации СП в таких технологических процессах в деформируемой заготовке должна быть предварительно получена мелкозернистая структура $[1,9,10,11-15]$, причем важно, чтобы температура СП обработки была по возможности низкой, что исключит необходимость специальной защиты от окисления и позволит использовать в качестве материала штампового инструмента не молибденовый, а никелевый сплав.

Физическая теория измельчения зерен при динамической рекристаллизации основывается на применении параметра Зинера-Холломона $Z$, который определяется в соответствии с уравнением $Z=\dot{\varepsilon} \cdot \exp (Q / R T)$, где $\dot{\varepsilon}-$ скорость деформации; $T$ - температура деформации; Q - энергия активации миграции границ зерен, $R$ - газовая постоянная. Чтобы получить меньший размер рекристаллизованных зерен, нужно увеличивать параметр $Z$, что достигается уменьшением $T$ и/или увеличением $\dot{\varepsilon}$. Очевидно, что для никелевых сплавов с высоким содержанием (>50 об.\%) упрочняющей $\gamma^{\prime}\left(\mathrm{Ni}_{3} \mathrm{Al}\right)$ фазы увеличение параметра Зинера-Холломона будет затруднено из-за ограниченной деформационной способности таких сплавов $[1,9,10]$. СП штамповка/раскатка высоколегированных никелевых сплавов при пониженных температурах также может быть затруднена, если нерастворенная дисперсная $\gamma^{\prime}$ фаза когерентно связана с матричной $\gamma$ фазой, что будет препятствовать развитию внутризеренного дислокационного скольжения и зернограничного проскальзывания - основного механизма СП деформации. Поэтому ДТО исходного компактированного сплава должна обеспечивать получение структуры дуплексного типа, когда размер упрочняющей $\gamma^{\prime}$ фазы сопоставим с размером $\gamma$ зерен, а межфазные $\gamma / \gamma^{\prime}$ границы не являются полностью когерентными [9-14].

Цель данной работы - исследование влияния режимов ДТО на микроструктуру и СП свойства порошкового никелевого сплава ЭП741НП. С практической точки зрения работа нацеливалась на определение температурно-скоростных условий СП штамповки и/или раскатки деталей типа диск для ГТД из сплава ЭП741НП, которые бы обеспечивали высокий выход годного металла.

\section{2. Материал и методики эксперимента}

Исходные заготовки сплава ЭП741НП $\varnothing 90 \times 60$ мм были изготовлены с помощью ГИП. Химический состав сплава (вес.\%): $\mathrm{Cr}-9.3 ; \mathrm{Ti}-1.8 ; \mathrm{Al}-5.2 ; \mathrm{Mo}-4$; $\mathrm{Nb}-2.7 ; \mathrm{Co}-15.7 ; \mathrm{W}-5.4 ; \mathrm{Hf}-0.2, \mathrm{C}-0.04, \mathrm{~B}-0.014$, $\mathrm{Ni}$ - основа. Температуру сольвуса $(T) \quad \gamma^{\prime}$ фазы определяли методом пробных закалок образцов от температур $T=1160-1200^{\circ} \mathrm{C}$, а также с помощью дифференциальной сканирующей калориметрии. Она составила $T_{s}=1180 \pm 5^{\circ} \mathrm{C}$. Исходную заготовку сплава подвергали гетерогенизационному отжигу (ГО) и ДТО, которая включала в себя трехстадийную одноосную деформацию сжатием в интервале температур $T=1140-1160^{\circ} \mathrm{C} \quad\left(\dot{\varepsilon} \sim 10^{-2} \mathrm{c}^{-1}\right) \quad$ в $\quad$ стальном контейнере $\mathrm{c}$ промежуточными рекристаллизационными отжигами на общую степень $e \approx 1.2$ (ДТО-1, состояние 1). Деформацию заготовок осуществляли на гидравлическом прессе с максимальным усилием $1600 \mathrm{Tc}$, температура штампового инструмента составляла $T=950^{\circ} \mathrm{C}$. Из одной полученной штамповки была вырезана заготовка, которую подвергали дополнительной однократной деформации сжатием в направлении, перпендикулярном направлению первой штамповки (ДТО-2, состояние 2). Деформацию осуществляли в изотермических условиях без контейнера при $T=950^{\circ} \mathrm{C}\left(\dot{\varepsilon} \sim 10^{-4}-10^{-3} \mathrm{c}^{-1}, e \approx 1.2\right)$, после чего заготовку отжигали при $T=900^{\circ} \mathrm{C}(12$ ч.).

Из полученных штамповок сплава вырезали плоские образцы с размером рабочей части $10 \times 5 \times 2$ мм³ $^{3}$. Испытания на растяжение при повышенных температурах были выполнены на воздухе с начальной скоростью деформации $\dot{\varepsilon}=5 \times 10^{-4}$ и $10^{-3} \mathrm{c}^{-1}$. 
Микроструктурные исследования деформированных заготовок проводили с центрального сечения, параллельного направлению последней штамповки c использованием сканирующей электронной микроскопии в режиме вторичных (SE) или обратнорассеянных электронов (BSE). Для мелкозернистых состояний сплава, а также для рабочей части образцов после растяжения в СП условиях был выполнен анализ дифракции обратно-рассеянных электронов (EBSD анализ) с шагом сканирования 0.1 мкм. EBSD анализ был выполнен с использованием программного обеспечения CHANNEL 5. Межзеренные и межфазные границы с разориентировкой менее $2^{\circ}$ были исключены из рассмотрения, принимая во внимание точность измерения. Высокоугловыми границами считали границы с углом разориентировки более $15^{\circ}$.

\section{3. Результаты и обсуждение}

\section{1. Микроструктура сплава}

В исходном состоянии (после ГИП) сплав имеет разнозернистую структуру, обусловленную спеканием порошинок разного размера. ГО приводит к коагуляции и сфероидизации частиц $\gamma^{\prime}$ фазы, при этом межфазные $\gamma / \gamma^{\prime}$ границы становятся полукогерентными или некогерентными [14]. Размер зерен $\gamma$ фазы после ГО не изменяется.

На Рис. S1 (дополнительный материал) представлены электронно-микроскопические (SE) изображения микроструктуры заготовок сплава в состояниях 1 и 2, а на Рис. S2 (дополнительный материал) ориентационные карты и спектр разориентировок межзеренных и межфазных границ, полученные с помощью EBSD анализа с центральной части заготовок. В состоянии 1 сплава достигнута в основном рекристаллизованная мелкозернистая структура дуплексного типа с преимущественно высокоугловыми границами зерен. Около $25 \%$ от общего числа границ составляют двойниковые границы зерен, что обусловлено быстрым развитием статической рекристаллизации. Этому способствует небольшой деформационный разогрев в процессе штамповки, a также медленное охлаждение деформированной заготовки в контейнере.

В состоянии 2 сплава, в отличие от состояния 1, формируется более мелкозернистая структура за счет образования областей с ультрамелкозернистой (УМЗ) структурой дуплексного типа $(d<1$ мкм), представляющая собой новые рекристаллизованные зерна $\gamma$ фазы, по границам и в тройных стыках которых присутствуют относительно крупные некогерентные или полукогерентные частицы $\gamma^{\prime}$ фазы. Результатом дополнительной деформационной обработки при пониженной температуре является также формирование развитой субструктуры в относительно крупных зернах, что в сочетании со значительно менее интенсивным развитием статической рекристаллизации после штамповки приводит к снижению доли высокоугловых границ (Табл. 1). Доля двойниковых границ уменьшается по сравнению с состоянием 1, что обусловлено пониженной температурой штамповки и относительно быстрым охлаждением деформированной заготовки сразу после завершения штамповки.

В Табл. 1 представлены количественные параметры микроструктуры сплава ЭП741НП в состояниях 1 и 2, полученные на основе совместного анализа электронномикроскопических изображений и ориентационных EBSD-карт. Средний размер $\gamma$ зерен в состоянии 1 сплава составляет $d=5.2$ мкм. В состоянии 2 сплава наблюдается два типоразмера $\gamma$ зерен: i) относительно крупные зерна размером $d=1.5-5$ мкм с развитой субструктурой внутри, рекристаллизованные в результате ДТО-1, но повторно нерекристаллизовавшиеся в процессе ДТО-2; ii) мелкие зерна со средним размером $d=0.7$ мкм, их объемная доля составляет $28 \%$. Следует отметить, что развитая субструктура в состоянии 2 сплава наблюдается не только в $\gamma$ зернах, но и в выделениях $\gamma^{\prime}$ фазы. В состоянии 1 сплава лишь в отдельных $\gamma$ зернах отмечается слабая субструктура.

Табл. 1. Параметры микроструктуры сплава ЭП741НП в состояниях 1 и 2.

Table 1. The microstructure characteristics of the EP741NP superalloy in conditions 1 and 2.

\begin{tabular}{|c|c|c|c|c|c|c|}
\hline \multirow{2}{*}{$\begin{array}{c}\text { Анализируемый параметр } \\
\text { Analyzed parameter }\end{array}$} & \multicolumn{6}{|c|}{$\begin{array}{c}\text { Количественные характеристики микроструктуры } \\
\text { Quantitative characteristics of the microstructure }\end{array}$} \\
\hline & \multicolumn{3}{|c|}{$\begin{array}{c}\text { Состояние } 1 \\
\text { Condition } 1\end{array}$} & \multicolumn{3}{|c|}{$\begin{array}{l}\text { Состояние } 2 \\
\text { Condition } 2\end{array}$} \\
\hline $\begin{array}{c}\text { Размер } \gamma \text { зерен, мкм } \\
\text { The } \gamma \text { grain size, } \mu \mathrm{m} \\
\end{array}$ & \multicolumn{3}{|c|}{5.2} & \multicolumn{3}{|c|}{$0.7 / 1.5-5$} \\
\hline $\begin{array}{c}\text { Объемная доля ультрамелкозернистых } \gamma \text { зерен, \% } \\
\text { The volume fraction of ultrafine } \gamma \text { grains, } \%\end{array}$ & \multicolumn{3}{|c|}{ - } & \multicolumn{3}{|c|}{28} \\
\hline $\begin{array}{l}\text { Размер частиц } \gamma^{\prime} \text { фазы, мкм } \\
\text { The size of the } \gamma^{\prime} \text { particles, } \mu \mathrm{m}\end{array}$ & 0.07 & 1.0 & 2.8 & 0.1 & $0.7-1.0$ & 2.9 \\
\hline $\begin{array}{l}\text { Объемная доля каждого типоразмера } \gamma^{\prime} \text { фазы, \% } \\
\text { The volume fraction of the } \gamma^{\prime} \text { phase of every size, \% }\end{array}$ & 35 & 5 & 17 & 5 & 32 & 20 \\
\hline $\begin{array}{c}\text { Доля высокоугловых границ, \% } \\
\text { The fraction of high-angle boundaries, \% }\end{array}$ & \multicolumn{3}{|c|}{89} & \multicolumn{3}{|c|}{62} \\
\hline $\begin{array}{c}\text { Доля двойниковых границ, \% } \\
\text { The fraction of twin boundaries, \% }\end{array}$ & \multicolumn{3}{|c|}{25.3} & \multicolumn{3}{|c|}{7.7} \\
\hline
\end{tabular}


В микроструктуре обоих состояний сплава выявляются три типоразмера $\gamma^{\prime}$ фазы: і) относительно крупные, нерастворившиеся при ДТО, частицы размером 2.8-2.9 мкм, занимающие объем 17-20\% и наблюдающиеся по границам $\gamma$ зерен; ii) частицы размером 0.7-1 мкм, их размер меньше, а объемная доля значительно выше в состоянии 2 сплава, чем в состоянии 1. Этот типоразмер $\gamma^{\prime}$ фазы обнаруживается в относительно крупных $\gamma$ зернах с развитой субструктурой, которая, по-видимому, способствует коагуляции дисперсной $\gamma^{\prime}$ фазы в процессе ДТО; iii) дисперсные выделения $\gamma^{\prime}$ фазы размером менее 0.1 мкм, выделившиеся при охлаждении заготовки после ДТО. Объемная доля дисперсных выделений значительна в состоянии 1 сплава (35\%), поскольку охлаждение штамповки осуществлялось с температур близких к температуре полного растворения $\gamma^{\prime}$ фазы, и незначительна (5\%) в состоянии 2 сплава, поскольку охлаждение заготовки после штамповки и последующего отжига происходило от сравнительно низкой температуры $\left(T=900-950^{\circ} \mathrm{C}\right)$.

Полученные результаты показывают, что при низкотемпературной деформационной обработке наряду c динамической рекристаллизацией интенсивно развивается динамический возврат, причем интенсивное внутризеренное скольжение развивается как в $\gamma$ зернах, так и в относительно крупных частицах $\gamma^{\prime}$ фазы. Формирование менее развитой субструктуры в частицах $\gamma^{\prime}$ фазы, в сравнении с $\gamma$ фазой объясняется, по всей видимости, большей прочностью и замедленностью процессов возврата в $\gamma^{\prime}$ фазе по сравнению с $\gamma$ фазой.

Таким образом, ДТО-2 приводит к измельчению структуры с образованием УМЗ составляющей благодаря развитию рекристаллизационных процессов при пониженной температуре, к формированию развитой субструктуры в относительно крупных $\gamma$ зернах и выделениях $\gamma^{\prime}$ фазы, к коагуляции $\gamma^{\prime}$ фазы и уменьшению доли двойниковых границ. Полученные штамповки сплава в состояниях 1 и 2 были использованы для изготовления образцов и последующего изучения СП свойств сплава.

\section{2. Сверхпластические свойства}

На Рис. S3 (дополнительный материал) представлены результаты испытаний образцов сплава в состояниях 1 и 2 на растяжение (при $\dot{\varepsilon}=10^{-3} \mathrm{c}^{-1}$ ). Видно, что кривые напряжение-деформация в случае обоих состояний характеризуются низким и почти постоянным уровнем напряжения течения при СП условиях, отвечающих максимальному удлинению. Для состояния 1 зависимость $\delta(T)$ имеет немонотонный характер с максимумом $(\delta=630 \%)$ при $T=1100^{\circ} \mathrm{C}$. Для состояния 2 удлинение монотонно возрастает в диапазоне $T=800-1000^{\circ} \mathrm{C}$, достигая наибольшего значения $(\delta=1320 \%)$ при $T=1000^{\circ} \mathrm{C}$ и $\dot{\varepsilon}=10^{-3} \mathrm{c}^{-1}$. Переход от состояния 1 к состоянию 2 приводит не только к снижению температуры проявления эффекта СП на $100^{\circ} \mathrm{C}$, но и к увеличению СП удлинений при сохранении низких напряжений течения $\left(\sigma_{30}=20-30 \mathrm{MПа}\right)$. Следует отметить, что СП свойства для обоих состояний оказались ниже в случае начальной скорости деформации $\dot{\varepsilon}=5 \times 10^{-4} \mathrm{c}^{-1}$.

\section{3. Микроструктурные изменения после сверхпластической дебормации}

На Рис. S4 (дополнительный материал) представлены электронно-микроскопические (SE) изображения, а на Рис. S5 (дополнительный материал) - ориентационные карты и спектр разориентировок межзеренных и межфазных границ, полученные с помощью EBSD анализа от рабочей части СП растянутых образцов сплава в состояниях 1 и 2. В Табл. 2 показаны количественные параметры микроструктуры сплава ЭП741НП в состояниях 1 и 2 после СП деформации.

Табл. 2. Параметры микроструктуры сплава ЭП741НП в состояниях 1 и 2 после СП деформации при $T=1100^{\circ} \mathrm{C}$ и $1000^{\circ} \mathrm{C}$, соответственно $\left(\dot{\varepsilon}=10^{-3} \mathrm{c}^{-1}\right)$.

Table 2. The microstructure characteristics of the EP741NP superalloy in conditions 1 and 2 after superplastic deformation at $T=1100^{\circ} \mathrm{C}$ and $1000^{\circ} \mathrm{C}$, respectively $\left(\dot{\varepsilon}=10^{-3} \mathrm{~s}^{-1}\right)$.

\begin{tabular}{|c|c|c|c|c|c|c|}
\hline \multirow{2}{*}{$\begin{array}{c}\text { Анализируемый параметр } \\
\text { Analyzed parameter }\end{array}$} & \multicolumn{6}{|c|}{$\begin{array}{c}\text { Количественные характеристики микроструктуры } \\
\text { Quantitative characteristics of the microstructure }\end{array}$} \\
\hline & \multicolumn{3}{|c|}{$\begin{array}{l}\text { Состояние } 1(\delta=630 \%) \\
\text { Condition } 1(\delta=630 \%)\end{array}$} & \multicolumn{3}{|c|}{$\begin{array}{l}\text { Состояние } 2(\delta=1320 \%) \\
\text { Condition } 2(\delta=1320 \%)\end{array}$} \\
\hline $\begin{array}{l}\text { Размер } \gamma \text { зерен, мкм } \\
\text { The } \gamma \text { grain size, } \mu \mathrm{m}\end{array}$ & \multicolumn{3}{|c|}{8.8} & \multicolumn{3}{|c|}{$1.3 / 1-5$} \\
\hline $\begin{array}{c}\text { Объемная доля ультрамелкозернистых } \gamma \text { зерен, \% } \\
\text { The volume fraction of ultrafine } \gamma \text { grains, \% }\end{array}$ & \multicolumn{3}{|c|}{-} & \multicolumn{3}{|c|}{35} \\
\hline $\begin{array}{l}\text { Размер частиц } \gamma^{\prime} \text { фазы, мкм } \\
\text { The size of the } \gamma^{\prime} \text { particles, } \mu \mathrm{m} \\
\end{array}$ & 0.2 & 1.0 & 3.0 & - & 1.0 & 2.9 \\
\hline $\begin{array}{l}\text { Объемная доля каждого типоразмера } \gamma^{\prime} \text { фазы, \% } \\
\text { The volume fraction of the } \gamma^{\prime} \text { phase of every size, \% }\end{array}$ & $30 \%$ & $7 \%$ & $20 \%$ & - & $36 \%$ & $21 \%$ \\
\hline $\begin{array}{c}\text { Доля высокоугловых границ, \% } \\
\text { The fraction of high-angle boundaries, \% }\end{array}$ & \multicolumn{3}{|c|}{77} & \multicolumn{3}{|c|}{85} \\
\hline $\begin{array}{c}\text { Доля двойниковых границ, \% } \\
\text { The fraction of twin boundaries, \% }\end{array}$ & \multicolumn{3}{|c|}{15} & \multicolumn{3}{|c|}{25.1} \\
\hline
\end{tabular}


Из сравнения Табл. 1 и 2 видно, что после СП деформации размер $\gamma$ зерен увеличивается в обоих состояниях. Это является результатом развития зернограничного проскальзывания [9]. После СП деформации образцов сплава ЭП741НП в состоянии 2 размер УМЗ $\gamma$ зерен тоже увеличивается, при этом их объемная доля несколько возрастает, что происходит, в том числе, за счет развития рекристаллизации в относительно крупных $\gamma$ зернах с развитой субструктурой. Доля высокоугловых границ несколько снижается в состоянии 1 за счет уменьшения доли двойниковых границ и увеличивается в состоянии 2 за счет, прежде всего, возрастания доли двойниковых границ, образовавшихся сразу после завершения испытания.

Размер крупных и средних выделений $\gamma^{\prime}$ фазы после СП деформации в состояниях 1 и 2 заметно не изменяется. Дисперсные выделения $\gamma^{\prime}$ фазы после СП деформации в состоянии 1 вырастают до 0.2 мкм, в состоянии 2 они не обнаружены, что объясняется, по-видимому, коагуляцией дисперсной $\gamma^{\prime}$ фазы в процессе СП деформации.

Таким образом, СП деформация приводит к некоторому росту $\gamma$ зерен в обоих состояниях сплава, однако в состоянии 2 рост $\gamma$ зерен происходит только в УМЗ составляющей структуры, в то время как в относительно крупнозернистой составляющей роста $\gamma$ зерен не наблюдается, а развиваются рекристаллизационные процессы, трансформирующие субструктуру в рекристаллизованную структуру с высокоугловыми границами зерен. Еще одна особенность изменения микроструктуры в состоянии 2 сплава в сравнении с состоянием 1 - это отмеченное выше исчезновение дисперсной $\gamma^{\prime}$ фазы, в то время как в состоянии 1 сплава дисперсная $\gamma^{\prime}$ фаза сохраняется, лишь увеличившись в размере до 0.2 мкм. Таким образом, снижение температуры проявления эффекта СП и улучшение СП характеристик в состоянии 2 сплава в сравнении с состоянием 1 объясняется не только увеличением протяженности высокоугловых межзеренных и межфазных границ благодаря формированию УМЗ составляющей структуры, но и достижением более термостабильной структуры, характеризующейся замедленным динамическим ростом $\gamma$ зерен в процессе СП течения. По-видимому, положительный вклад в достижение повышенных удлинений в состоянии 2 сплава оказывает и уменьшение доли двойниковых границ в сравнении состоянием 1 , поскольку известно, что они неблагоприятны для развития зернограничного проскальзывания.

Представленные данные показывают возможность снижения температуры проявления эффекта СП и повышения СП удлинений в сплаве ЭП741НП после дополнительной ДТО при пониженной температуре, обеспечившей формирование смешанной мелкозернистой структуры со значительной объемной долей УМЗ составляющей. Измельчение $\gamma$ зерен и $\gamma^{\prime}$ частиц способствует развитию при низких температурах зернограничного проскальзывания, чей вклад оказывается значительным за счет высокой протяженности межзеренных и межфазных границ.
Вместе с тем, повышенные СП удлинения, достигнутые в состоянии 2 сплава при $T=1000^{\circ} \mathrm{C}$, по всей вероятности, обусловлены также значительно большим содержанием нерастворившейся $\gamma^{\prime}$ фазы, чем в состоянии 1 при $T=1100^{\circ} \mathrm{C}$, причем размер частиц нерастворившейся $\gamma^{\prime}$ фазы в состоянии 2 сплава близок к размеру $\gamma$ зерен. Объемная доля УМЗ $\gamma$ зерен $(d=0.7$ мкм) в состоянии 2 сплава составляет $28 \%$, а объемная доля частиц $\gamma^{\prime}$ фазы с размером 0.7-1 мкм $32 \%$, т.е. 60 об.\% микроструктуры составляет структура дуплексного типа с близким размером $\gamma$ зерен и $\gamma^{\prime}$ частиц, равным 0.7-1 мкм (Табл. 1). Объемная доля более крупных частиц $\gamma^{\prime}$ фазы со средним размером 2.9 мкм занимает около $20 \%$, а доля $\gamma$ зерен с размером 1.5-5 мкм занимает оставшиеся 20 об.\% (Табл. 1). Таким образом, более крупнозернистая составляющая структуры в состоянии 2 сплава также имеет дуплексный тип с близким размером $\gamma$ зерен и $\gamma^{\prime}$ частиц, равным 1.5-5 мкм. Этим объясняется высокая термостабильность микроструктуры в состоянии 2 сплава в процессе СП течения, которая способствует его устойчивости и достижению высоких удлинений. Напротив, состояние 1 сплава при температуре $1100^{\circ} \mathrm{C}$ содержит лишь около 20 об.\% $\gamma^{\prime}$ фазы, сопоставимой по размеру с $\gamma$ зернами, что обеспечивает меньшую термостабильность структуры (Табл. 1 и 2).

Таким образом, в результате ДТО-2 в сплаве была получена структура дуплексного типа с размером $\gamma$ зерен/ $\gamma^{\prime}$ частиц в диапазоне 0.7-5 мкм, которая обеспечивает необычно высокую стабильность микроструктуры в процессе СП течения. Вместе с высокой протяженностью межзеренных и межфазных границ это способствует увеличению вклада зернограничного проскальзывания в деформацию и достижению высоких СП удлинений при пониженных температурах.

\section{4. Заключение}

В работе исследованы СП свойства порошкового никелевого сплава ЭП741НП, подвергнутого различной ДТО. Установлено, что дополнительная ДТО при пониженной температуре формирует наиболее благоприятную для проявления эффекта СП структуру дуплексного типа с размером $\gamma$ зерен $/ \gamma^{\prime}$ частиц в диапазоне $d=0.7-5$ мкм. Образцы сплава в полученном состоянии демонстрируют пониженную температуру проявления эффекта СП (близкую к температуре старения этого сплава) при одновременно повышенных СП удлинениях в сравнении с относительно крупнозернистым состоянием, полученным в результате ДТО при более высокой температуре.

Микроструктурный анализ деформированных образцов показал, что высокие СП удлинения обусловлены не только высокой протяженностью межзеренных и межфазных границ, обеспечившей значительный вклад в деформацию зернограничного проскальзывания - основного механизма СП деформации, но и необычно высокой стабильностью микроструктуры - слабым изменением размера $\gamma$ зерен и $\gamma^{\prime}$ частиц в процессе СП течения. 
Дополнительныйматериал/Supplementarymaterial. Электронная версия статьи содержит дополнительный материал, доступный безвозмездно на сайте журнала (lettersonmaterials.com)./The online version of this paper contains supplementary material available free of charge at the journal's Web site (lettersonmaterials.com).

Благодарности/Aknowledgements. Работа выполнена при поддержке Российского научного фонда (грант РНФ № 18-19-00685) и в рамках Госзадания ИПСМ РАН (№ АААА-А17-117041310215-4). Экспериментальные исследования были выполнены на базе Центра коллективного пользования научным оборудованием ИПСМ РАН. / The present work was supported by the Russian Science Foundation (Grant No.18-19-00685) and the State Assignment of the Institute for Metals Superplasticity Problems of the Russian Academy of Sciences (No. AAAA-A17-117041310215-4). The work was performed using the facilities of the shared services center "Structural and Physical-Mechanical Studies of Materials" at the Institute for Metals Superplasticity Problems of Russian Academy of Sciences.

\section{Литература/References}

1. S.T. Kishkin. Sozdaniye, issledovaniye i primeneniye zharoprochnykh splavov: izbrannyye trudy (K 100-letiyu so dnya rozhdeniya). Moscow, Nauka (2006) 407 p. (in Russian) [C. Т. Кишкин. Создание, исследование и применение жаропрочных сплавов: избранные труды (К 100-летию со дня рождения). Москва, Наука (2006) 407 c.]

2. A.F. Belov, N.F. Anoshkin, O. Kh. Fatkullin. Struktura i svoystva granuliruyemykh nikelevykh splavov. Moscow, Metallurgiya (1984) 127 p. (in Russian) [А.Ф. Белов, Н.Ф. Аношкин, О.Х. Фаткуллин. Структура и свойства гранулируемых никелевых сплавов. Москва, Металлургия (1984) 127 с.]

3. A. V. Vostrikov, G.S. Garibov. Gas turbine technology. 3 (46), 34 (2006). (in Russian) [А.В. Востриков, Г.С. Гарибов. Газотурбинные технологии. 3 (46), 34 (2006).]
4. R. L. Athey, J. B. Moore. National Aerospace Engineering and Manufacturing meeting. Los Angeles, USA (1975). pp. 1-11.

5. Yu. A. Nozhnitskiy. Technology of light alloy. 3, 13 (2007). (in Russian) [Ю.А. Ножницкий. Технология легких сплавов. 3, 13 (2007).]

6. S. A. Kononov, A.S. Perevozov, B. A. Kolachev. Metally. 5, 86 (2007). (in Russian) [C. А. Кононов, А. С. Перевозов, Б. А. Колачев. Металлы. 5, 86 (2007).]

7. O.Kh. Fatkullin, V.I. Eremenko, O.N. Vlasova, N.M. Grits. Technology of light alloy. 5-6, 149 (2001). (in Russian) [O.Х. Фаткуллин, В.И. Еременко, О.Н. Власова, Н. М. Гриц. Технология легких сплавов. 5-6, 149 (2001).]

8. O.Kh. Fatkullin, V.I. Eremenko, O.N. Vlasova, V.G. Sklyarenko. Technology of light alloy. 4, 105 (2002). (in Russian) [О.Х. Фаткуллин, В.И. Еременко, О.Н. Власова, В.Г. Скляренко. Технологии легких сплавов. 4, 105 (2002).]

9. O.A. Kaibyshev, F.Z. Utyashev. Superplasticity: Microstructural Refinement and Superplastic Roll Forming. Futurepast. Arlington, Virginia, USA (2005). 386 p.

10. R. R. Mulyukov, R.M. Imayev, A.A. Nazarov et al. Sverkhplastichnost' ul'tramelkozernistykh splavov: eksperiment, teoriya, tekhnologii. Moscow, Nauka (2014) 284 p. (in Russian). [Р. Р. Мулюков, Р. М. Имаев, А. А. Назаров и др. Сверхпластичность ультрамелкозернистых сплавов: эксперимент, теория, технологии. Москва, Наука (2014) 284 с.]

11. O. A. Kaibyshev, V. A. Valitov, G. A. Salishchev. The Physics of Metals and Metallography. 4, 110 (1993).] (in Russian) [О.А. Кайбышев, В. А. Валитов, Г. А. Салищев. Физика металлов и металловедение. 4, 110 (1993).]

12. V.A. Valitov. Letters on Materials. 3(1), 50 (2013). (in Russian) [В.А. Валитов. Письма о материалах. 3(1), 50 (2013).] Crossref 13. V.A. Valitov, G.A. Salishchev, Sh.Kh. Mukhtarov. Materials Science Forum. 243 - 245, 557 (1997) Crossref 14. A. A. Ganeev, V.A. Valitov. Letters on Materials. 5 (2), 152 (2015). (in Russian) [А.А. Ганеев, В.А. Валитов. Письма о Материалах. 5 (2), 152 (2015).] Crossref

15. V. A. Valitov, G. A. Salishchev, Sh. Mukhtarov. Metally. 3, 127 (1994). (in Russian) [В. А. Валитов, Г. А. Салищев, Ш.Х. Мухтаров. Металлы. 3, 127 (1994).] 